
U0qUr

\author{
Adriane D.M. Vorselaars ${ }^{1}$, Heleen A. Crommelin ${ }^{1,2}$, Coline H.M. van Moorsel ${ }^{1,3}$ and Jan C. Grutters ${ }^{1,3}$ \\ ${ }^{1}$ Interstitial Lung Diseases Centre of Excellence, Dept of Pulmonology, St Antonius Hospital, Nieuwegein, The \\ Netherlands. ${ }^{2}$ Dept of Clinical Pharmacy, St Antonius Hospital, Nieuwegein, The Netherlands. ${ }^{3}$ Division of Heart and
} Lungs, University Medical Centre Utrecht, Utrecht, The Netherlands.

Correspondence: Jan C. Grutters, Interstitial Lung Diseases Centre of Excellence, Dept of Pulmonology, St Antonius Hospital, Koekoekslaan 1, 3435 CM Nieuwegein, The Netherlands. E-mail: j.grutters@antoniusziekenhuis.nl

Received: Aug 282015 | Accepted: Sept 012015

Conflict of interest: None declared.

\title{
References
}

1 Vorselaars ADM, Crommelin HA, Deneer VHM, et al. Effectiveness of infliximab in refractory FDG PET-positive sarcoidosis. Eur Respir J 2015; 46: 175-185.

2 Statement on sarcoidosis. Joint Statement of the American Thoracic Society (ATS), the European Respiratory Society (ERS) and the World Association of Sarcoidosis and Other Granulomatous Disorders (WASOG) adopted by the ATS Board of Directors and by the ERS Executive Committee, February 1999. Am J Respir Crit Care Med 1999; 160: 736-755

3 Harris J, Keane J. How tumor necrosis factor blockers interfere with tuberculosis immunity. Clin Exp Immunol 2010; 161: 1-9.

4 World Health Organization. Global tuberculosis report 2015. Geneva, WHO Press 2015

5 Drake WP, Newman LS. Mycobacterial antigens may be important in sarcoidosis pathogenesis. Curr Opin Pulm Med 2006; 12: 359-363.

6 Newman LS, Rose CS, Bresnitz EA, et al. A case control etiological study of sarcoidosis: environmental and occupational risk factors. Am J Respir Crit Care Med 2004; 170: 1324-1330.

7 Martens H, Zöllner B, Zissel G, et al. Anti-Borrelia burgdorferi immunoglobulin seroprevalence in pulmonary sarcoidosis: a negative report. Eur Respir J 1997; 10: 1356-1358.

8 Ebe Y, Ikushima S, Yamagichi T, et al. Proliferative response of peripheral blood mononuclear cells and levels of antibody to recombinant protein from Propionibacterium acnes DNA expression library in Japanese patients with sarcoidosis. Sarcoidosis Vasc Diffuse Lung Dis 2000; 17: 256-265.

9 Eishi Y, Suga M, Ishige E, et al. Quantative analysis of mycobacterial and propionibacterial DNA in lymph nodes of Japanese and European patients with sarcoidosis. J Clin Microbiol 2002; 40: 198-204.

10 Müller-Quernheim J, Prasse A, Zissel G. Pathogenesis of sarcoidosis. Presse Med 2012; 41: e275-e287.

11 Crommelin HA, Vorselaars ADM, van Moorsel CHM, et al. Anti-TNF therapeutics for the treatment of sarcoidosis. Immunotherapy 2014; 6: 1127-1143.

\section{Haemoptysis: a frequent diagnostic challenge}

\section{To the Editor:}

We read with interest the article by ABDULMALAK et al. [1] recently published in the European Respiratory Journal. The authors reported the results of an observational, retrospective, 5-year, nationwide, multicentre study based on the medical information collected from a French database. The epidemiology of haemoptysis was evaluated through the hospital discharge diagnosis codes, focusing on incidence, aetiology, seasonal distribution, relapses and mortality in a 3-year follow-up analysis. The authors made a great effort to provide findings on the largest national cohort of hospitalised patients with haemoptysis ( $\sim 15000$ per year) and update the current epidemiological understanding of this frequent symptom in high-income countries. However, some of the results described in the manuscript deserve a more detailed analysis and careful interpretation.

The authors found that cryptogenic or idiopathic haemoptysis, defined as haemoptysis without an established cause [2, 3], was the most frequent aetiology ranging from $48.9 \%$ in 2012 to $52 \%$ in 2008 . Although the epidemiology of haemoptysis has changed in the last decades, these data are significantly different to those described in other European hospitalised cohorts. In two prospective series, idiopathic haemoptysis had an incidence range of $5.4-13 \%[2,4]$, whilst three retrospective studies detected an 
incidence of $6.3-33.7 \%$ [5-7]. Notably, another study in which the epidemiological retrospective design was based on discharge codes described a higher rate of idiopathic bleeds (42.2\%) [8].

It is also worth noticing that a significant percentage of idiopathic haemoptysis reached a definite diagnosis during a recurrence. In both 2008 and 2009, about $10 \%$ of patients initially diagnosed with a cryptogenic or a respiratory infection-related haemoptysis were subsequently diagnosed with lung cancer in a follow-up period of 3 years. Of note, about half of these new cases of malignancies were diagnosed within only 2 months after the initial bleeding episodes. The only European study that prospectively assessed the course of inpatients with idiopathic haemoptysis described no lung cancer cases during a 4-year follow-up [2].

In our opinion, these findings may have two potential explanations: the lack of an accurate initial diagnostic workup and/or the questionable use of the administrative coding system to explore the epidemiology of a symptom. Cryptogenic haemoptysis was defined as an incident case without any database aetiologies coded as the main/associated/related diagnosis. Moreover, the same authors acknowledged that the haemoptysis code may have been used inappropriately, probably more often in cases requiring large amounts of care. The initial high percentage of idiopathic and infection-related haemoptysis subsequently diagnosed as lung cancer within only 2 months might be associated with a misleading approach related to a laboratory delay. Nevertheless, the discrepancy with other epidemiological reports confirms that the epidemiological approach adopted by ABDULMALAK et al. [1] might have significantly biased the results. We deem that observational prospective or retrospective studies should include more information sources, including clinical files or hospital discharge letters.

Haemoptysis is a challenging symptom frequently involving life-threatening conditions, such as lung cancer. The aetiology of haemoptysis may sometimes be misled by an incomplete initial diagnosis: the diagnostic work-up should be as exhaustive as possible, particularly if malignancy risk factors are recognised [9]. Despite its clinical relevance, optimal diagnostic workup remains largely unclear. There is a lack of evidence-based guidelines regarding appropriate diagnostic management of patients with haemoptysis; the majority of the studies, mostly retrospective, show poor evidence on the best initial panel of tests [3-9]. This condition may lead to inaccurate diagnosis and to avoidable radiation exposure and invasive tests. Future prospective studies should clarify these issues, providing an essential diagnostic pathway according to the accuracy of the main tests (i.e. chest radiography, computed tomography and bronchoscopy), amount of bleeding, demographic and epidemiological data.

A prospective, Italian, multicentre trial (www.ClinicalTrials.gov identifier NCT02045394) is currently underway, focusing on the description of the epidemiology of haemoptysis in both in- and outpatients, and on the identification of an essential diagnostic algorithm.

Future studies, based on a more accurate epidemiological design, could better describe the causes of haemoptysis and contribute to fill the gaps concerning the diagnosis and management of this symptom.

0 @ERSpublications

Haemoptysis is a frequent symptom, and the definition of aetiologies and a diagnostic pathway are often challenging http://ow.ly/U0f2X

Michele Mondoni ${ }^{1}$, Giuseppe Francesco Sferrazza Papa ${ }^{1,2}$, Giovanni Sotgiu ${ }^{3}$, Paolo Carlucci ${ }^{1}$, Giulia Michela Pellegrino ${ }^{1}$ and Stefano Centanni ${ }^{1}$

${ }^{1}$ Respiratory Unit, San Paolo Hospital, Dept of Scienze della Salute, Università degli Studi di Milano, Milan, Italy. ${ }^{2}$ Casa di Cura del Policlinico, Dipartimento di Scienze Neuroriabilitative, Milan, Italy. ${ }^{3}$ Clinical Epidemiology and Medical Statistics Unit, Dept of Biomedical Sciences, University of Sassari-Research, Medical Education and Professional Development Unit, AOU Sassari, Sassari, Italy.

Correspondence: Giuseppe Francesco Sferrazza Papa, Respiratory Unit, San Paolo Hospital, Dept of Scienze della Salute, Università degli Studi di Milano, Via A. Di Rudinì 8, 20142 Milan, Italy. E-mail: francesco.sferrazza@gmail.com

Received: Aug 132015 | Accepted: Aug 192015

Conflict of interest: None declared.

\section{References}

1 Abdulmalak C, Cottenet J, Beltramo G, et al. Haemoptysis in adults: a 5-year study using the French nationwide hospital administrative database. Eur Respir J 2015; 46: 503-511.

2 Savale L, Parrot A, Khalil A, et al. Cryptogenic haemoptysis from a benign to a life-threatening pathologic vascular condition. Am J Respir Crit Care Med 2007; 175: 1181-1185.

3 Herth F, Ernst A, Becker HD. Long-term outcome and lung cancer incidence in patients with haemoptysis of unknown origin. Chest 2001; 120: 1592-1594.

4 Tsoumakidou M, Chrysofakis G, Tsiligianni I, et al. A prospective analysis of 184 haemoptysis cases - diagnostic impact of chest X-ray, computed tomography, bronchoscopy. Respiration 2006; 73: 808-814. 
5 Soares Pires F, Teixeira N, Coelho F, et al. Haemoptysis - etiology, evaluation and treatment in a university hospital. Rev Port Pneumol 2011; 17: 7-14.

6 Fartoukh M, Khoshnood B, Parrot A, et al. Early prediction of in-hospital mortality of patients with haemoptysis: an approach to defining severe haemoptysis. Respiration 2012; 83: 106-114.

7 Khalil A, Soussan M, Mangiapan G, et al. Utility of high-resolution chest CT scan in the emergency management of haemoptysis in the intensive care unit: severity, localization and etiology. Br J Radiol 2007; 80: 21-25.

8 Boulay F, Berthier F, Sisteron O, et al. Seasonal variation in cryptogenic and noncryptogenic haemoptysis hospitalizations in France. Chest 2000; 118: 440-444.

9 Thirumaran M, Sundar R, Sutcliffe IM, et al. Is investigation of patients with haemoptysis and normal chest radiograph justified? Thorax 2009; 64: 854-856.

Eur Respir J 2016; 47:348-350 | DOI: 10.1183/13993003.01344-2015 | Copyright @eERS 2016

\section{From the authors:}

We appreciate and agree with the comments of M. Mondoni and colleagues, that the results of the ongoing prospective Italian multicentre trial (www.ClinicalTrial.gov identifier NCT02045394) will be of considerable interest for the management of haemoptysis, a frequent and severe symptom, especially since there is no clear consensus concerning aetiology and treatment. Interestingly, beyond determining the prevalence of diseases that may present with haemoptysis, their epidemiological results will be analysed according to the severity of the symptom. More importantly, their trial will hopefully make it possible to analyse the sensitivity and specificity of complementary tests, such as chest radiography, chest computed tomography (CT) and bronchoscopy alone and in combination in the diagnosis of different causes of haemoptysis.

We acknowledged that our observational retrospective study had some limitations and possible bias due to the way data was collected, that is to say through hospital discharge diagnosis codes [1]. However, the national administrative database (PMSI/medicalisation of information system programme) has gathered national administrative health data in France from every french public and private teaching and nonteaching healthcare facility since 1997. Each hospital's budget depends on the medical activity, as described by a specific computer program, which compiled discharge abstracts related to all admissions since 2008. The fact that these national data are necessary to allocate hospital budgets has considerably encouraged improvements in data quality in terms of accuracy and exhaustiveness, and the results obtained in our study are consequently different from those in the publication of Boulay et al. [2], where data were collected between 1994 and 1997. In France, diagnoses identified during hospital stays have been coded according to the 10th edition of the International Classification of Diseases (ICD-10) since 1998. In ICD-10, there is a single specific code for haemoptysis (ICD-10 code R042). Therefore, and importantly, we are confident that the symptom was not over- or misreported in our study.

Our database did not allow us to collect data from out-patients, who may only have presented mild haemoptysis. There is therefore a possible risk that we over-selected moderate and severe haemoptysis and missed some cases of mild haemoptysis [3]. Physicians may also have underreported mild haemoptysis in patients with a previously known diagnosis of lung cancer when the patient was hospitalised with this symptom, but not due to it. The aetiology of haemoptysis may have been reported with a delay, and we believe this is particularly the case for lung cancer. Indeed, because it may take some time to gather results from other complementary examinations or pathology reports, the patient may have been discharged from the initial hospitalisation without a diagnosis of lung cancer, even though the physician suspected the disease. To report lung cancer in the PMSI, it is necessary to have definite pathological confirmation of the diagnosis. However, we were aware of this possible bias and considered that lung cancer was the aetiology of the initial haemoptysis episode when it was diagnosed within 2 months of the initial haemoptysis. We therefore discussed the hypothesis that lung cancer developed in $4 \%$ of patients with initial cryptogenic haemoptysis during the 3 years of follow-up [1]. This hypothesis is in accordance with previously published studies [4].

Despite these unavoidable biases and possible inaccuracies due to the coding system, we believe that our 5 -year study provided unequivocal and important data regarding the 10 million patients who are hospitalised yearly in France, among whom 15000 (0.2\%) were admitted for haemoptysis or have haemoptysis as a complication of their hospital stay [1]. We provided important information regarding prognosis and follow-up. We showed that the frequency of recurrence was relatively low, since $84 \%$ of patients with initial haemoptysis had no recurrence within the 3 years of follow-up. Moreover, even though it was explored in small prospective studies, we found substantial mortality rates during the initial stay as expected [5] but, more interestingly, during the follow-up as well. Indeed, the 3-year in-hospital mortality rate, excluding lung cancers, was high at $20 \%$. Even if death may have been more closely related 